\title{
Optical-trapping micromanipulation using 780-nm diode lasers
}

\author{
T. C. Bakker Schut, E. F. Schipper, B. G. de Grooth, and J. Greve \\ Cell Characterization Group, Department of Applied Physics, University of Twente, 7500 AE Enschede, The Netherlands
}

Received October 5, 1992

\begin{abstract}
We have designed and implemented an optical-trapping configuration that uses near-infrared laser diodes. The highly divergent output beam of the diode laser was collimated by using only one aspheric compact disc lens. The resulting output beams are astigmatic and elliptic and have a flat, non-Gaussian intensity profile. Calculations and measurements were performed to investigate the influence of this profile on the trapping forces. The results show that use of a laser diode, collimated with a compact disc lens, provides a near-infrared light source that can be used for optical trapping. The light source is compact and relatively cheap and can be easily incorporated into an existing microscope.
\end{abstract}

Optical trapping provides a new way for micromanipulation of small objects like cells, as has been demonstrated by Ashkin et al. ${ }^{1}$ The use of such optical tweezers has the advantage that one can even manipulate within cells without damaging the cell membrane. ${ }^{2}$ The light sources most often used for optical tweezers are Nd:YAG and argon-ion lasers. Recently, diode lasers have also been used ${ }^{3,4}$ to build a relatively compact micromanipulation system that can be easily incorporated into a microscope. ${ }^{4}$

We investigated the possibility of using a singlemode 780-nm laser diode as a light source for optical trapping. The emitted light is still visible, which is easy for alignment and observation without an imaging system, while the absorption for both cells and surrounding aqueous solutions is negligible. ${ }^{5}$ The output beam of the diode laser was collimated by using only one aspheric lens, which is also used in compact disc (CD) players. The resulting intensity profile is not Gaussian but more-or-less flat. The influence of the intensity profile on the trapping forces was calculated by using a geometrical-optics model described earlier. ${ }^{6}$ The calculations show that this intensity profile could give rise to an increased stability in the direction of light propagation and a more limited trapping capacity in the direction orthogonal to it, as has been recently shown for apertured Gaussian TEM T0 $_{00}$ beams by Ashkin. ${ }^{7}$ Measurements of the trapping forces along the beam axis on polystyrene spheres in a single-beam trap were performed to verify the calculations. Measurements of the maximum manipulation speed in the direction orthogonal to the beam axis were done for lymphocytes in a vertical double-beam trap.

The diode lasers used were 780-nm single-mode gain-guided GaAlAs diode lasers with a maximum output power of $40-50 \mathrm{~mW}$ (Sony SLD201). The two orthogonal divergence angles (FWHM) of the laser beam are typically approximately $28^{\circ}$ (perpendicular to the active layer of the laser diode) and approximately $14^{\circ}$ (parallel to the active layer). The beam is astigmatic, with the distance between the two beam waists $\left|Z_{\|}-Z_{\perp}\right|$ typically $40 \mu \mathrm{m}$. The beams were collimated by using Philips CD lenses $(f=$ $4.5 \mathrm{~mm}, \mathrm{~N} . \mathrm{A} .=0.45$ ). These CD lenses are aspheric and specially designed to focus highly divergent laser beams. ${ }^{8} \quad$ A schematic drawing of the laser diode in combination with the CD lens is given in Fig. 1. Because of the astigmatism, the laser beam cannot be collimated completely. The output beam after the lens is elliptic (diameters $w_{\|} \cong 1.9 \mathrm{~mm}$ and $w_{\perp} \cong$ $3.9 \mathrm{~mm}$ ). The intensity profiles in the two orthogonal directions, shown in Fig. 2, are not Gaussian, but more flat-top shaped. A more Gaussian profile can be obtained by placing a diaphragm between the laser diode and the CD lens, but this greatly reduces the intensity of the beam and is therefore undesirable.

A geometrical-optics model, described earlier, ${ }^{6}$ was used to calculate the forces that are exerted by a focused Gaussian laser beam on a trapped particle. The influence of the non-Gaussian intensity profile of the laser beams was investigated by calculating the forces that would be exerted by a beam with a Gaussian propagation but with a flat-top intensity profile. The intensity profile of the collimated diode laser is somewhat in between a Gaussian and a flattop profile.

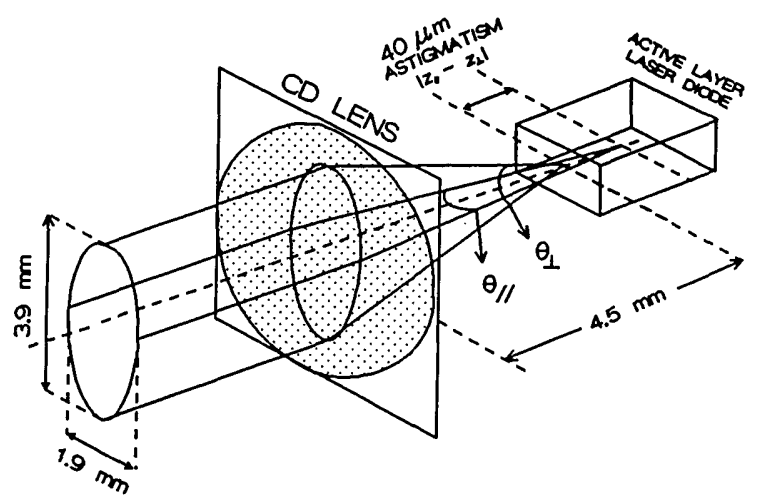

Fig. 1. Schematic of the laser diode and the CD lens used for collimation. 


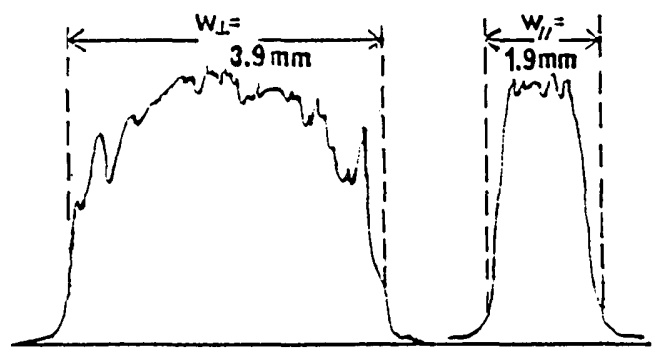

Fig. 2. Intensity profiles in the two directions orthogonal $\left(w_{\perp}=3.9 \mathrm{~mm}\right)$ and parallel $\left(w_{\|}=1.9 \mathrm{~mm}\right)$ to the active layer of the laser diode after collimation by using a CD lens.

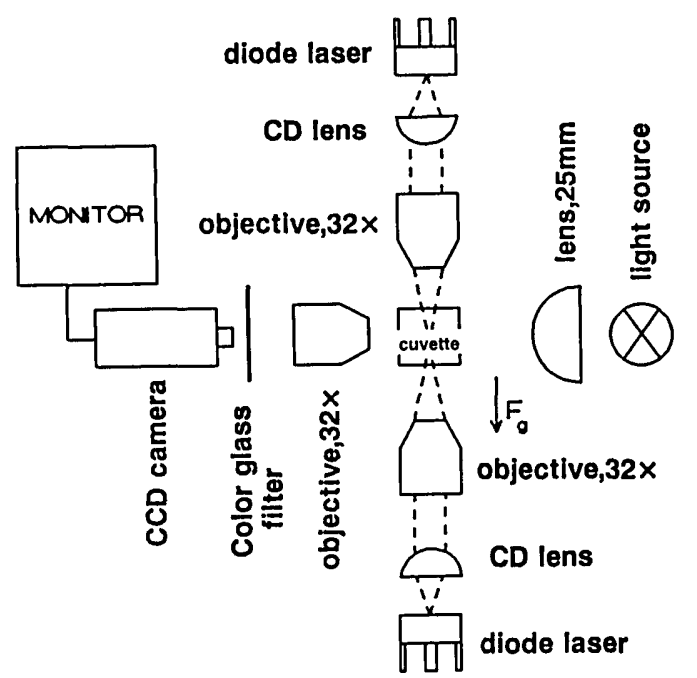

Fig. 3. Schematic of the experimental setup.

The trapping forces were measured by using both a vertical single-beam gravity trap and a vertical double-beam trap (using two diode lasers). A schematic of the configuration used is shown in Fig. 3. The collimated diode laser light was focused to a spot with a radius of $1.5 \mu \mathrm{m}\left(r_{\|}\right)$by using a Leitz 32×, 0.40-N.A. objective (Leitz GmbH, Wetzlar, Germany). The spheres were suspended in distilled water and contained in a quartz cuvette (Hellma $\mathrm{GmbH}$, Müllheim, Germany). The cuvette can be positioned by using encoder stepper mikes (Model 18011, Oriel Corporation, Stratford, Connecticut).

The position of the particles inside the cuvette was monitored with a Panasonic F10 CCD camera (Matsushita Electronics). The size of the image on the monitor was calibrated with a microscope calibration target and the stepper mikes (resolution $<0.5 \mu \mathrm{m}$ ) so that the position of the spheres relative to the focus could be measured. Measurements were recorded with an S-VHS video recorder (JVC HR-S5000E). The power of the laser light inside the cuvette was calculated by measuring at points in front of and behind the cuvette with a power monitor (Newport Model 835, Fountain Valley, California) and correcting for the losses at the transitions.

Polystyrene spheres with a mean diameter of $9.6 \mu \mathrm{m}$ (Polysciences, Inc., Warrington, Pennsylvania), refractive index of $1.58,{ }^{9}$ and specific gravity of $1.059 \times 10^{3} \mathrm{~kg} / \mathrm{m}^{3}$ were used to determine the optical force $F_{Z}$ along the beam axis in a singlebeam trap. $F_{Z}$ was measured by determining the equilibrium positions in the gravity trap as a function of the power $p$ of the incident light, $F_{Z}(p)=F_{G}$, and then calculating the value of $F_{Z}$ at a power of $1 \mathrm{~W}: F_{Z}(1)=F_{Z}(p) / p$. This method is limited to the regions where $\partial F_{Z}(z) / \partial z<0$, since only there we can have an equilibrium. ${ }^{10}$ The maximum possible error in the measurement of $F_{Z}$ when using this method is estimated to be $10 \%$.

Human lymphocytes were used to determine the maximum micromanipulation speed $v_{\max }$ in the direction orthogonal to the beam axis. When moving, the friction (Stokes) force is in equilibrium with the force $F_{Y}(z, y)$ that the light exerts on the particle in the direction toward the beam axis, and the force $F_{Z}(z, y)$ is in equilibrium with the gravity force $F_{G}$. Therefore, the conditions for attaining the maximum speed are given by

$$
\begin{aligned}
& F_{Y, \max }(z, y)=F_{\text {Stokes }}\left(v_{\max }\right)=6 \pi \eta r v_{\max }, \\
& F_{Z, \max }(z, y)=F_{G},
\end{aligned}
$$

where $\eta$ is the viscosity of water $\left(10^{-3} \mathrm{~N} \mathrm{~s} \mathrm{~m}^{-2}\right)$ and $r$ is the radius of the particle. The maximum error in the measurement of $v_{\max }$ is estimated to be $30 \%$.

In order to investigate the forces along the beam axis, both calculations and measurements were performed by using homogeneous polystyrene spheres with a diameter of $9.6 \mu \mathrm{m}$. In Fig. 4 the calculated and the measured forces are shown. Calculations were performed for both a Gaussian and a flat-top intensity profile of (1-W) incident laser light. The figure shows that the flat-top profile theoretically gives rise to an increased stability in the direction of propagation of light. The measured forces are somewhat higher than the calculated ones but show the same dependence on the distance to the focus. In order to determine the theoretical maximum manipulation speed, we calculated the forces in a vertical double-beam optical trap for both Gaussian and flat-top intensity profiles. The focus of the upward

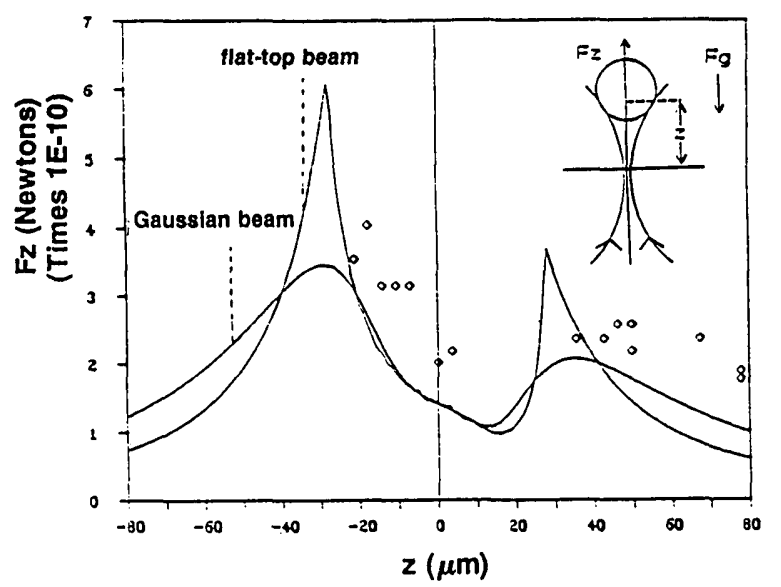

Fig. 4. Calculated and measured forces on a homogeneous polystyrene sphere (radius $=4.8 \mu \mathrm{m}$, relative refractive index $=1.6$ ) along the beam axis in a single-beam optical trap for a Gaussian and a flat-top intensity profile. Beam waist $=3 \mu \mathrm{m}$. Measurements (scaled to a laser power of $1 \mathrm{~W}$ ) are indicated as rhombs. 


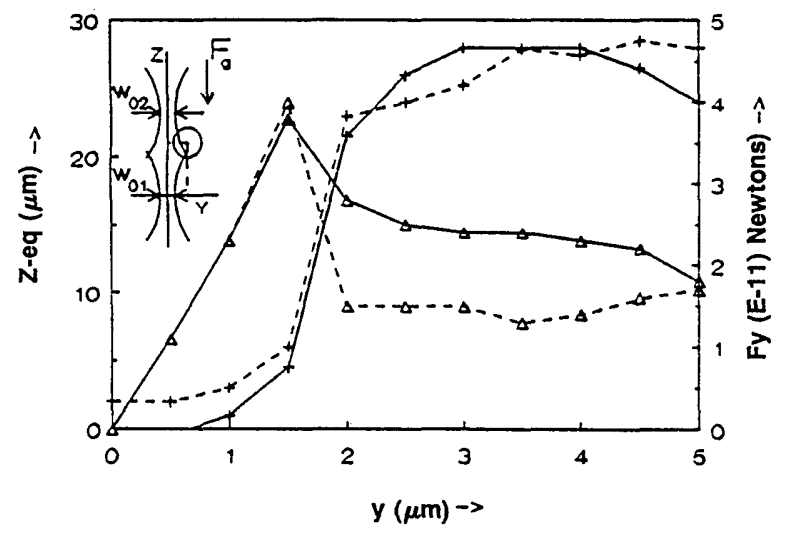

Fig. 5. Equilibrium position ( $Z$-eq) and the force toward the beam axis $\left(F_{Y}\right)$ functions of the distance $Y$ from the beam axis in a double-beam trap for a lymphocyte (radius $=3.5 \mu \mathrm{m}$, relative refractive index $=1.05$, specific gravity $=1071 \mathrm{~kg} / \mathrm{m}^{3}$ ). $w_{01}=w_{02}=3 \mu \mathrm{m}$. The foci are $70 \mu \mathrm{m}$ apart in the $z$ direction and are both centered at $y=0$. Crosses, $z$ values of the equilibrium positions (right axis); triangles, $F_{Y}$ forces (left axis); solid curves, Gaussian intensity profile; dashed curves, flat-top intensity profile.

beam is $70 \mu \mathrm{m}$ below the focus of the downward beam. For both beams $16 \mathrm{~mW}$ of laser power was used. Cells were initially trapped in the middle of the two foci. In the calculations a lymphocyte was modeled as a homogeneous sphere with a relative refractive index of $1.05 .^{11}$ The calculated gravitational net force for a lymphocyte $7 \mu \mathrm{m}$ in diameter with a specific gravity of $1071 \mathrm{~kg} / \mathrm{m}^{3}$ is $1.3 \times 10^{-13} \mathrm{~N}$. Figure 5 shows the calculated values $F_{Y}$ and the corresponding $z$ positions for which $F_{Z}$ is in equilibrium with $F_{G}$ as a function of the distance $y$ from the beam axis. From Fig. 5 , it can be seen that for a Gaussian intensity profile the maximum $F_{Y}$ force is approximately $3.8 \times 10^{-12} \mathrm{~N}$, corresponding to a velocity of approximately $58 \mu \mathrm{m} / \mathrm{s}$ using Eqs. (1). For a flat-top intensity, the maximum force is even slightly higher, i.e., $4 \times 10^{-12} \mathrm{~N}$, corresponding to a velocity of approximately $61 \mu \mathrm{m} / \mathrm{s}$. Measurements on a lymphocyte in a vertical double-beam trap show that these cells can be manipulated with speeds of approximately $30 \mu \mathrm{m} / \mathrm{s}$ in the direction orthogonal to the beam axis.

The measurements of the forces on polystyrene spheres along the beam axis in a single-beam trap are in reasonable agreement with the calculations, as can be seen from Fig. 4 . The measured values are somewhat higher than the calculated values but the measurements show the same dependence on $z$ as the calculated values. The differences between the calculated and measured forces might be caused by the fact that the experimental conditions do not really justify the use of a geometrical-optics model; $2 \pi r / \lambda=2 \pi\left(4.8 \times 10^{-6}\right) /\left(780 \times 10^{-9}\right)=38.7$. Earlier experiments (with $r=3.75 \mu \mathrm{m}$ and $\lambda=$ $488 \mathrm{~nm}$ ) showed the same tendencies. ${ }^{6}$ Figure 4 shows that a flat-top intensity profile gives rise to increased maximum forces and an increased stability (determined by the slope of the stable region) in the direction of propagation of light. The influence of the
non-Gaussian intensity profile on the trapping force could not be measured; the difference between the two theoretical models is small compared with the difference between the theory and the measurements.

The measurements of the manipulation speed orthogonal to the beam axis in a double-beam trap are somewhat lower than the calculated maximum speed. Apart from the fact that the model is also not valid in this case, it must be noted that the measurements of the maximum speed (and thus $F_{Y}$ ) are limited by the fact that the encoder mikes that were used do not accelerate slowly but impose the preset velocity on the particle immediately. The initial acceleration imposes another force on the particle. This is especially critical in case one wants to measure the maximum $F_{Y}$ force, because then there is no equilibrium in the beginning, which may cause the cell to slip out of the force field of the beam. Therefore the maximum practical manipulation speed will always be lower than the calculated speed. From Fig. 5 it can be seen that if the beginning acceleration is too high, the cell exceeds $y=1.5$ and the resulting $F_{Y}$ forces will be smaller, i.e., $2.5 \times 10^{-12} \mathrm{~N}$ for the Gaussian intensity profile and $1.5 \times 10^{-12} \mathrm{~N}$ for the flat-top profile. The corresponding manipulation speeds are 38 and $22 \mu \mathrm{m} / \mathrm{s}$ for the Gaussian and the flat-top profiles, respectively. These speeds are in much better agreement with the experimentally determined maximum manipulation speeds.

In conclusion, the output beams of 780-nm laser diodes, collimated only with a CD lens, can be used for optical trapping. The experimentally determined forces are in reasonable agreement with the calculations, using a geometrical-optics model. Differences between calculations and measurements are probably due to the limited validity of the geometrical model for the experimental conditions. From the measurements it cannot be concluded which of the two intensity profiles is the best description for the actual profile.

\section{References}

1. A. Ashkin, J. M. Dziedzic, and T. Yamane, Nature (London) 330, 769 (1987).

2. S. M. Block, D. F. Blair, and H. C. Berg, Biophys. J. 55, 258a (1989).

3. S. Sato, M. Ohyumi, H. Shibata, and I. Inaba, Opt. Lett. 16, 282 (1991).

4. R. S. Afzal and E. B. Treacy, Rev. Sci. Instrum. 63, 2157 (1992).

5. T. Kasuya and M. Tsukakushi, Laser Microirradiation of Cells (Harwood Academic, Chur, Switzerland, 1988), pp. 11-13.

6. T. C. Bakker Schut, G. Hesselink, B. G. de Grooth, and J. Greve, Cytometry 12, 497 (1991).

7. A. Ashkin, Biophys. J. 61, 569 (1992).

8. J. J. M. Braat, A. Smid, and M. M. B. Wijnakker, Appl. Opt. 24, 1853 (1985).

9. D. E. Gray, ed., American Institute of Physics Handbook, 3rd ed. (McGraw-Hill, New York, 1972), p. 6-109.

10. A. Ashkin, Appl. Phys. Lett. 24, 586 (1974).

11. P. M. A. Sloot, A. G. Hoekstra, and C. G. Figdor, Cytometry 9, 636 (1988). 